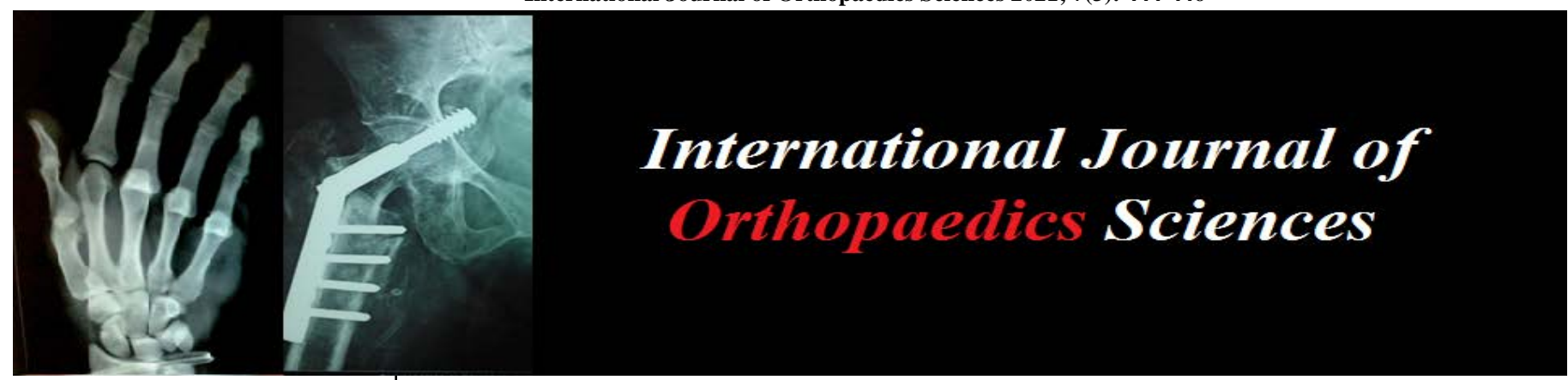

E-ISSN: 2395-1958

P-ISSN: 2706-6630

IJOS 2021; 7(3): 444-446

(C) $2021 \mathrm{IJOS}$

www.orthopaper.com

Received: 07-05-2021

Accepted: 09-06-2021

Dr. Jyothis George

MBBS, M.S. Ortho, DNB Ortho,

Assistant Professor

Department of Orthopedics

Government Medical College,

Kottayam, Kerala, India

Dr. Anandu Mathews Anto MBBS, Orthopedic Resident Department of Orthopedics Government Medical College Kottayam, Kerala, India
Corresponding Author: Dr. Jyothis George MBBS, M.S. Ortho, DNB Ortho, Assistant Professor

Department of Orthopedics Government Medical College,

Kottayam, Kerala, India

\section{Mini open non vascularized fibula graft harvesting technique without any specialized instrumentation: A case report}

\section{Dr. Jyothis George and Dr. Anandu Mathews Anto}

DOI: https://doi.org/10.22271/ortho.2021.v7.i3g.2784

\section{Abstract}

Fibula graft harvesting is a common procedure in orthopedic practice. The common approach to the shaft of fibula is through long incision made over the fibula. This was fraught with complications including common peroneal nerve injuries, pain and significant graft site morbidity to patients. Techniques then came to describe harvesting fibula through minimal incisions. However these needed specialized circular harvesters and hence was difficult to perform in hospitals catering to poor socioeconomic strata. We have devised minimally invasive technique of fibula harvesting using a long 4.5 system narrow DCP (Dynamic compression plate) used as soft tissue stripper and have combined the procedure with mini open fasciotomy and compression dressing. Our patient did not have any postoperative common peroneal, superficial peroneal or deep peroneal nerve injury. Postoperative graft site pain was minimal.

Keywords: fibular grafting, free fibular graft, minimal invasive fibular graft

\section{Introduction}

Case presentation

A 35 year old post covid gentleman with nonunion comminuted supracondylar intercondylar fracture femur underwent open reduction and internal fixation using distal femoral LCP (Locking compression plate).A fibular strut graft was planned for this patient in view of severe medial side comminution ${ }^{[1]}$.

\section{Technique of Fibular graft harvesting}

The approximate length of fibula required is identified. The middle third of fibula sparing the proximal $10 \mathrm{~cm}$ and distal $10 \mathrm{~cm}$ (upto a maximum of $8 \mathrm{~cm}$ from ankle joint) ${ }^{[2]}$ is selected as the site of graft harvesting. Skin marking are made of the lateral aspect of leg at the site of proximal and distal fibula osteotomy Proximal and distal skin incisions of approximately $1 \mathrm{~cm}$ is made over the posterolateral aspect of fibula (Fig 1). A fasciotomy of the lateral compartment is made through this mini incision for preventing compartment syndrome ${ }^{3}$ and pain due to post op edema (Fig 2). The plane is identified between the soleus muscles posteriorly and the peroneus muscles anteriorly. The fibula is identified at the proximal and distal ends and multiple drill holes are made at the site of proximal and distal osteotomy. And the osteotomy is made complete with an osteotome. A 4.5 system narrow DCP of appropriate length is taken and soft tissue around the fibula is detached (Fig 3). Laterally, anteriorly posteriorly. The fibula is held at its one end using a bone holder and the medial side or the interosseous side is detached off the interosseous ligament using the 4.5 system DCP. The fibula is rotated within the wound to check whether any soft tissue tether is remaining (Fig 4). And the fibula is delivered out through the mini incision (Fig 5). Immediately, compression dressing is applied.

Post-operative period once the effect of subarachnoid block has weaned thorough neurological examination was done to look for evidence of foot drop or paresthesia. There was full power ankle dorsiflexion and no evidence of soft tissue collection on change of dressing. 


\section{Follow-up}

Patient was assessed on post-operative day 3 , on postoperative day 14 and at 6 weeks postoperatively. Postoperative x-ray was taken on day 3 (Fig 6) and graft site wounds appeared clean and dry (Fig 7).The patient had a visual analogue score of 3 when asked about graft site discomfort on post-operative day 3, VAS score of 1 on post-operative day 14 and no discomfort at 6 weeks postop(VAS 10 as maximum discomfort and 0 as no discomfort).Patient did not have graft site pain, minimal cosmetic complaints, ankle motion was supple with full ankle and big toe dorsiflexion (Fig8), no evidence of deep vein thrombosis, no complaints of sensory or motor weakness.

\section{Discussion}

Fibula graft harvesting which was conventionally performed through an open approach cannot justify the post procedure graft site morbidity. Hence the concept of mini open fibula grafting came into practice. The common issues facing the mini open fibulectomy are

1. Lack of instrumentation like a specialized circular stripper ${ }^{[1 a]}$.

2. Damage to peroneal artery and bleeding from graft site leading to compartment syndrome.

3. Lack of knowledge regarding the technique

4. Using periosteal elevator creates difficulty in accessing the middle half of the graft segment in case of long segment harvesting.

5. Using periosteal elevator will strip the graft off of periosteum, which makes the graft biologically inferior

\section{The factors favoring this technique include}

1. Minimal soft tissue injury

2. Low possibility of extensor hallucis longus weakness ${ }^{[4]}$

3. Cosmetically better

4. Less chance of infection

5. Less chance of injury to superficial peroneal nerve or sural nerve

6. Earlier return to work

7. Less chance of periosteum getting stripped off.

8. Using a long DCP plate as the soft tissue separator will overcome the difficulty of accessing the middle half of a long graft segment

9. The mini fasciotomy helps to reduce swelling and pain due to the swelling

\section{Figures}

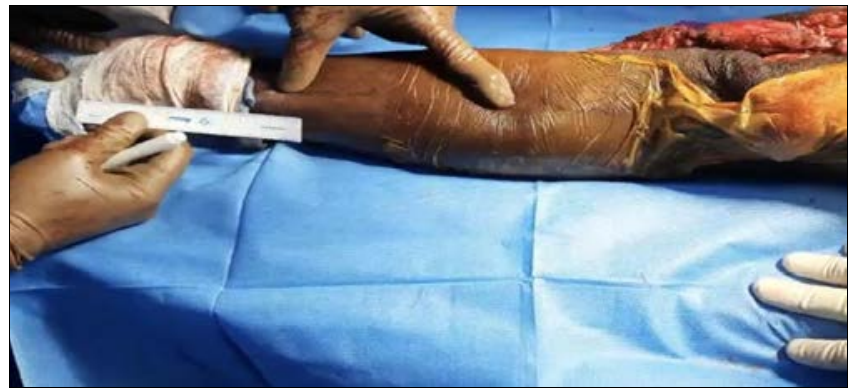

Fig 1: Planning of the skin incision at the site of proximal and distal fibular osteotomy

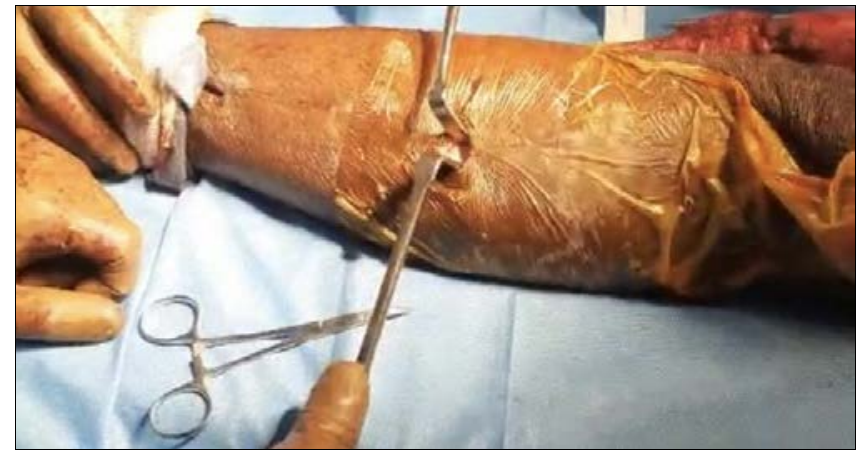

Fig 2: Proximal and distal skin incisions made and mini fasciotomy of lateral compartment

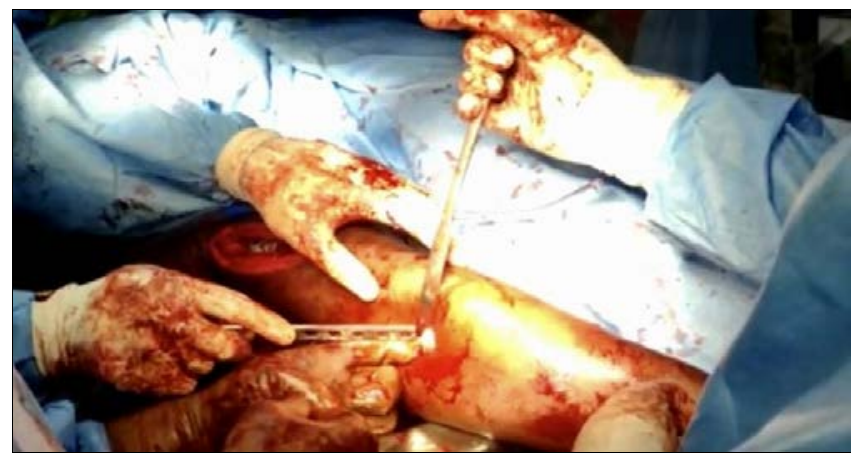

Fig 3: Fibula identified and 4.5 system narrow DCP is used as soft tissue stripper.

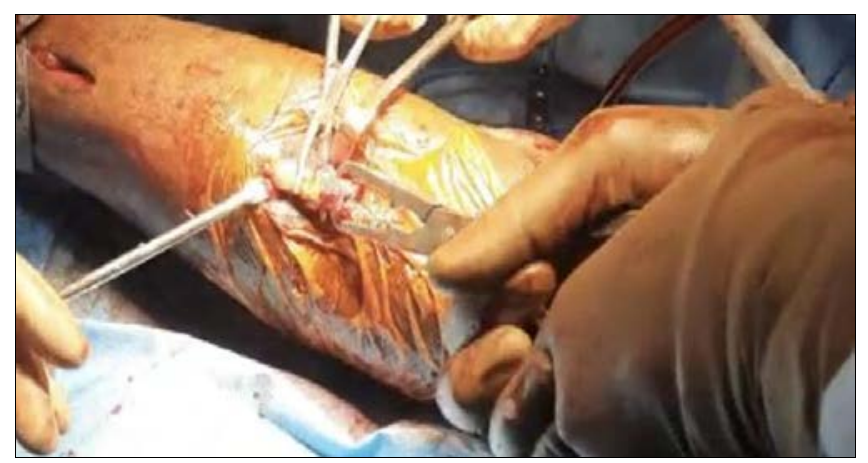

Fig 4: Fibula held at one end using a bone holder and rotated while delivering the fibula through the mini incision.

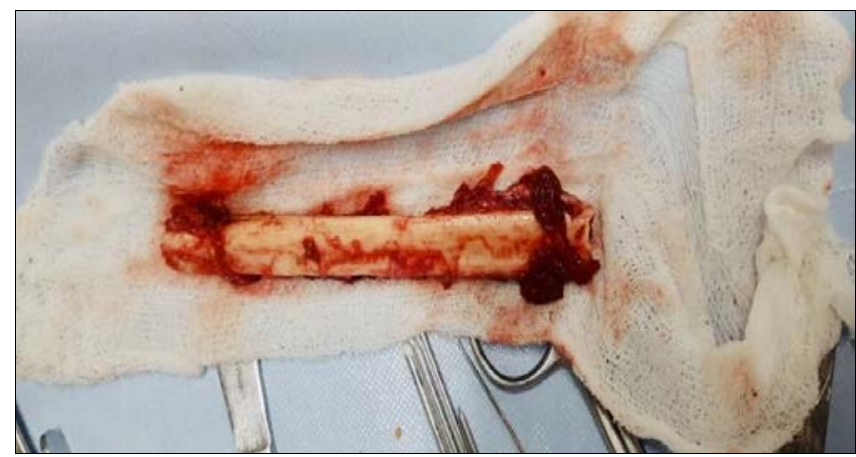

Fig 5: The extracted fibula 


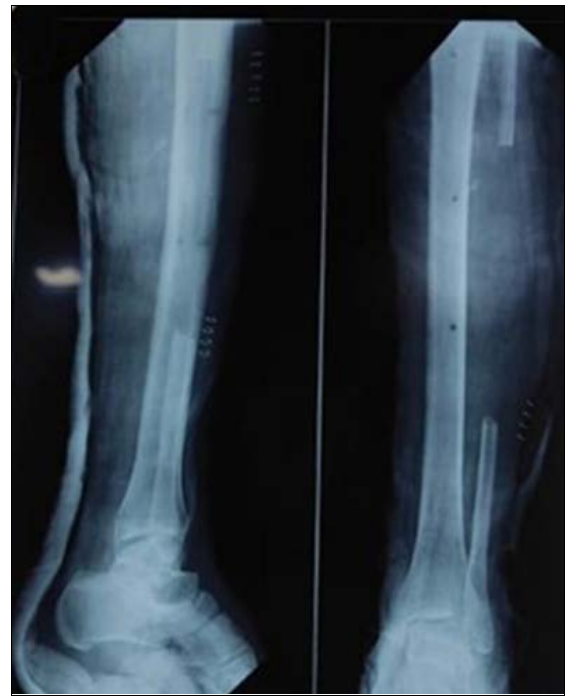

Fig 6: Immediate postop x-ray of fibulectomy site

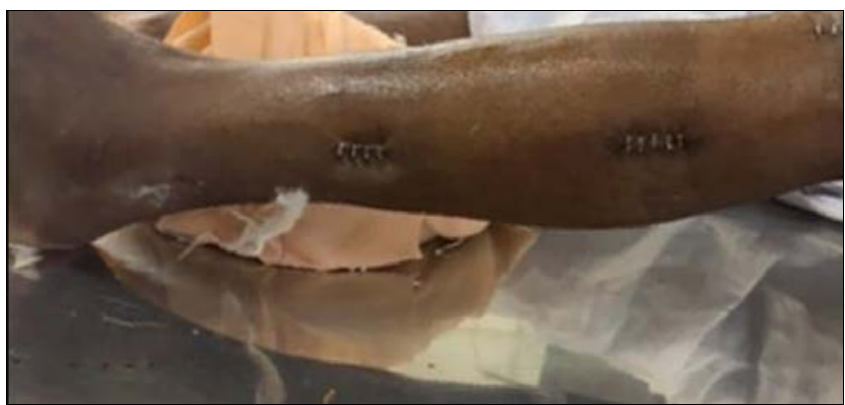

Fig 7: Graft site on postop day 3 appears clean, dry and healthy.

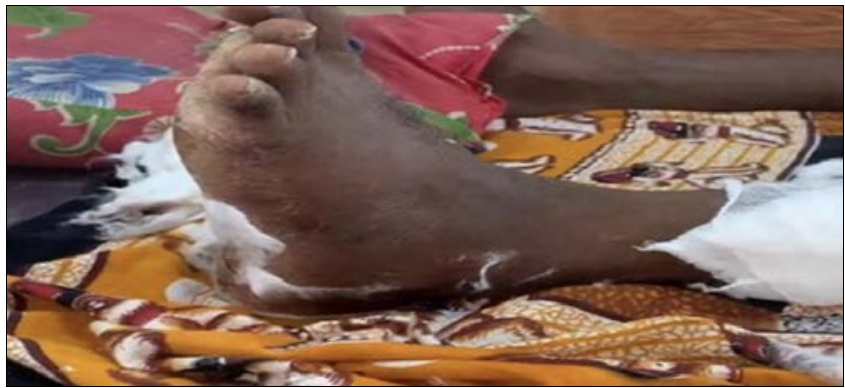

Fig 8: Postop day 3 -ankle dorsiflexion intact when assessed on postoperative day 3 .

\section{References}

1. Mukherjee AN, Pal AK, Singharoy D, Baksi D, Nath C. Harvesting the free fibular graft: A modified approach. Indian J Orthop 2011;45(1):53-6.

2. Pacelli LL, Gillard J, McLoughlin SW, Buehler MJ. A Biomechanical Analysis of Donor-Site Ankle Instability Following Free Fibular Graft Harvest. JBJS 2003;85(4):597-603.

3. S00590-021-02919-z.pdf [Internet]. [Cited 2021 Jul 28]. Available from: https://link.springer.com/content/pdf/10.1007/s00590021-02919-z.pdf.

4. Lucas G, Lopez J, Fraisse B, Marleix S, Violas P. Minimally invasive harvesting of non-vascularized fibular graft in children. Orthopaedics \& Traumatology: Surgery \& Research 2015;101(4):515-8. 